\title{
Quizartinib (AC220) is a potent second generation class III tyrosine kinase inhibitor that displays a distinct inhibition profile against mutant-FLT3, -PDGFRA and -KIT isoforms
}

Kerstin Maria Kampa-Schittenhelm" ${ }^{*}$, Michael Charles Heinrich², Figen Akmut ${ }^{1}$, Hartmut Döhner ${ }^{3}$, Konstanze Döhner ${ }^{3}$ and Marcus Matthias Schittenhelm

\begin{abstract}
Background: Activating mutations of class III receptor tyrosine kinases (RTK) FLT3, PDGFR and KIT are associated with multiple human neoplasms including hematologic malignancies, for example: systemic mast cell disorders (KIT), non-CML myeloproliferative neoplasms (PDGFR) and subsets of acute leukemias (FLT3 and KIT). First generation tyrosine kinase inhibitors (TKI) are rapidly being integrated into routine cancer care. However, the expanding spectrum of TK-mutations, bioavailability issues and the emerging problem of primary or secondary TKl-therapy resistance have lead to the search for novel second generation TKls to improve target potency and to overcome resistant clones.

Quizartinib was recently demonstrated to be a selective FLT3 inhibitor with excellent pharmacokinetics and promising in vivo activity in a phase II study for FLT3 ITD + AML patients. In vitro kinase assays have suggested that in addition to FLT3, quizartinib also targets related class III RTK isoforms.
\end{abstract}

Methods: Various FLT3 or KIT leukemia cell lines and native blasts were used to determine the antiproliferative and proapoptotic efficacy of quizartinib. To better compare differences between the mutant kinase isoforms, we generated an isogenic BaF3 cell line expressing different FLT3, KIT or BCR/ABL isoforms. Using immunoblotting, we examined the effects of quizartinib on activation of mutant KIT or FLT3 isoforms.

Results: Kinase inhibition of (mutant) KIT, PDGFR and FLT3 isoforms by quizartinib leads to potent inhibition of cellular proliferation and induction of apoptosis in in vitro leukemia models as well as in native leukemia blasts treated ex vivo. However, the sensitivity patterns vary widely depending on the underlying (mutant)-kinase isoform, with some isoforms being relatively insensitive to this agent (e.g. FLT3 D835V and KIT codon D816 mutations). Evaluation of sensitivities in an isogenic cellular background confirms a direct association with the underlying mutant-TK isoform - which is further validated by immunoblotting experiments demonstrating kinase inhibition consistent with the cellular sensitivity/resistance to quizartinib.

Conclusion: Quizartinib is a potent second-generation class III receptor TK-inhibitor - but specific, mutation restricted spectrum of activity may require mutation screening prior to therapy.

Keywords: AC220, Quizartinib, Leukemia, KIT, FLT3, PDGFR

\footnotetext{
* Correspondence: kerstin.kampa-schittenhelm@med.uni-tuebingen.de

'Department of Hematology, Oncology, Rheumatology, Immunology and

Pulmology, University Hospital Tübingen, Tübingen, Germany

Full list of author information is available at the end of the article
} 


\section{Background}

Gain-of-function mutations of the FLT3, KIT and PDGFR class III receptor tyrosine kinases (RTK) play important roles as oncogenesis-driving events in several hematologic malignancies. For example, FLT3 mutations are predominantly found in AML associating with a poor prognosis [1-4], but are also reported in (pediatric) acute lymphoblastic leukemia (ALL) [5]. KIT mutations occur in the vast majority of systemic mastocytosis (SM) [6] and subsets of acute leukemia, including corebinding factor (CBF) [7] and pediatric [8] AML. Certain FLT3 and KIT mutations correlate with inferior outcome in adult AML $[4,9,10]$.

PDGFR mutations are frequently found in myeloproliferative disorders, such as Philadelphia chromosomenegative chronic myeloid leukemia (CML), where PDGFR alpha or beta fuses with another gene allowing autoactivation of the tyrosine kinase. Several fusion partners have been described, including FIP1L1 leading to the FIP1L1-PDGFRA fusion gene. This translocation has been associated with hypereosinophilic syndromes and mastocytosis with eosinophilia [11-13].

Numerous tyrosine kinase inhibitors have been developed to target class III RTKs (see also Discussion). These TKIs have a variable spectrum of activity against different class III RTKs and against various mutant isoforms of these kinases. To date, translation from bench to bedside has resulted in only modest or shortlived effectiveness of these inhibitors in most entities [14-23] and only a few agents have achieved FDAapproval for selected indications such as CML and HES. With the exception of $\mathrm{Ph}+\mathrm{ALL}$, no TKIs have been approved for treatment of acute leukemia so far.

Quizartinib is a novel second generation class III receptor tyrosine kinase inhibitor with superior pharmaceutical properties and an excellent pharmacokinetic profile compared to other agents. Quizartinib was demonstrated to have high efficacy and tolerability in tumor xenograft models that express a FLT3 ITD mutant kinase $[24,25]$.

A previous study used recombinant enzyme in in vitro kinase assays to identify that quizartinib targets related class III RTKs, such as wildtype and gain-of-function mutant KIT and PDGFR isoforms [24].

Using several cell based assays, we now show, that quizartinib treatment of leukemic cells leads to inhibition of mutant KIT, PDGFR and FLT3 isoforms - with resultant inhibition of cellular proliferation and induction of apoptosis. These effects are seen in vitro as well as ex vivo (primary leukemic blasts). Importantly, potent antitumor activity was seen against distinct (mutated) kinase isoforms, including FIP1L1-PDGFRA, and FLT3 ITD, FLT3 TKD1 and FLT3 TKD2 mutations. Whereas some mutant-KIT and -FLT3 isoforms were sensitive to quizartinib treatment, some mutations such as FLT3 D835V and the most prevalent KIT gain-of-function mutation detected in CBF AML, KIT D816V, was relatively insensitive with regard to quizartinib treatment.

Quizartinib is currently under clinical investigation in FLT3 ITD and wildtype AML. Our data suggests that quizartinib may be an attractive agent for clinical investigation in other settings as outlined here. This would not include the group of mutant-KIT CBF AML that have KIT D816V mutations. However, patients with CBF AML with KIT D816Y or exon 11 mutations or patients with solid tumors associated with KIT and PDGFR mutations, such as GIST might benefit from this agent. Clinical mutation analysis could help identify individuals that are the most likely to respond to quizartinib.

\section{Results}

Quizartinib inhibits cellular proliferation of mutant-FLT3, KIT or-PDGFRA leukemia cell lines in a dose dependent manner

Quizartinib was previously reported to be a potent inhibitor of wildtype FLT3 and FLT3 ITD kinases [24]. Structural considerations suggest quizartinib could inhibit other members of the class III RTK family that are frequently mutated in leukemia or myeloproliferative disorders (i.e. KIT and PDGFR). These findings prompted us to evaluate quizartinib sensitivity in a variety of leukemia cell line models harboring RTK mutations.

The human mast cell leukemia cell lines HMC1.1 (KIT V560G) and HMC1.2 (KIT V560G + D816V), the murine mast cell line p815 (harboring a KIT D814Y mutation analogous to the human D816Y mutation), the eosinophilic leukemia cell line EOL-1 (FIP1L1-PDGFRA), the CBF AML cell line Kasumi-1 (N822K), the myeloid leukemia cell line MOLM14 (heterozygous FLT3 ITD), M-07e (growth factor dependent wildtype KIT), the APL cell line HL60 (growth factor independent, wildtype FLT3 and KIT), the lymphoblastic leukemia cell line Jurkat (no known activated RTK) and the CML blast crisis cell line K562 (BCR/ABL1) were treated with quizartinib in a dose-dependent manner for 48 hours and the cellular antiproliferative capacity was measured using an XTT-based assay.

The proliferation of cell lines with FLT3 ITD (MV4;11, MOLM14), FIP1L1-PDGFRA (EOL-1), ligand-stimulated wild-type KIT (M0-7e), or certain KIT mutations (KIT exon 11 V560G, HMC1.1; Kasumi KIT exon 17 N822K) was strongly inhibited by quizartinib (Figure 1). In contrast, the proliferation of a cell line with a KIT exon 11 V560G and KIT exon 17 D816V mutation on the same allele (HMC1.2) was basically insensitive to quizartinib. Noteworthy, the murine cell line p815, harboring an alternative (tyrosine) substitution at the same codon (KIT 

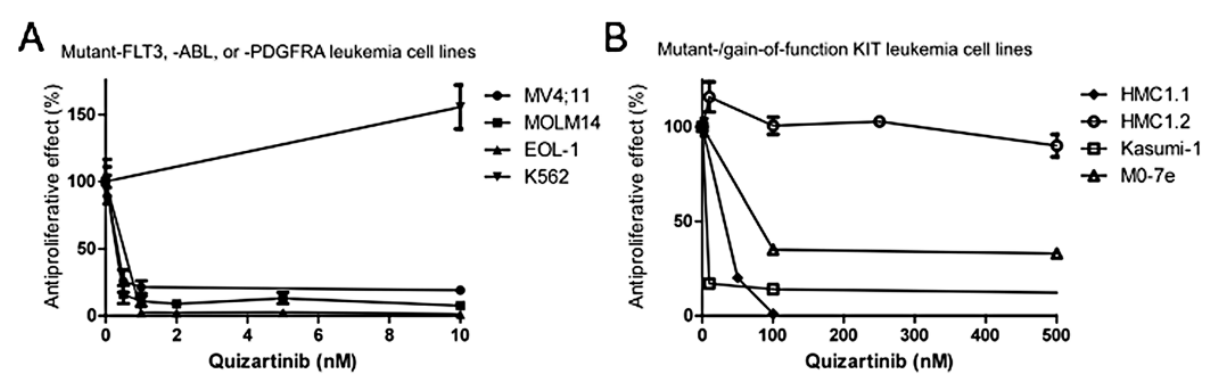

Figure 1 Quizartinib inhibits cellular proliferation of KIT-, FLT3- or PDGFR-dependent leukemia cells. Dilution series of quizartinib for several cell lines harboring mutant-FLT3, ABL1 or PDGFRA (1A) or mutant-KIT isoforms (1B) were performed and cellular proliferation was assessed using an XTT-based assay. Sensitivity towards quizartinib varied widely between the tested cell lines. IC50s and mutation isoforms for all tested cell lines are listed in Table 1.

D814Y, which corresponds to D816Y in human KIT), retained intermediate sensitivity to quizartinib.

As experimental controls, we also tested quizartinib against several cell lines lacking an activated type III RTK (K562 [BCR-ABL1], Jurkat [no known tyrosine kinase oncogene], and HL60 [no known tyrosine kinase oncogene, but expressed wild-type FLT3]; quizartinib had no meaningful anti-proliferative effects against these cell lines (IC50 > 10,000 nM; Table 1).

DMSO alone, used in the highest concentration in any of the quizartinib dosing experiments, had no significant antiproliferative effect (MOLM14 cell line, Table 1).

To further show the specificity of the anti-proliferative effect of quizartinib, we tested the effects of this drug against M-07e cultured in GM-CSF rather than SCF: Whereas the SCF-stimulated M-07e were sensitive to quizartinib (IC50 $77 \mathrm{nM}$ ), GM-CSF stimulated M-07e were completely resistant (quizartinib IC50 > 10,000 nM).

Notably, the sensitivity patterns observed for different mutant RTK isoforms were not totally consistent with IC50s previously published in a kinase assay reported by Zarrinkar and colleagues [24] (e.g. IC50 ${ }_{\mathrm{KIT}}$ D816V 150 $\mathrm{nM}$ versus $>1500 \mathrm{nM}$ in our assays). There are several possible explanations for this disparity. First, the results by Zarrinkar et al. were performed using drug binding to recombinant kinases as opposed to enzymatic inhibition of full-length cellular kinases. Second, while we did not change FBS levels prior to quizartinib administration ( $10 \%$ for leukemia cell lines, $20 \%$ for native leukemia blasts) - the earlier report by Zarrinkar et al. [24] used cells that were pre-sensitized by exposure to reduced serum levels (0.5\% FBS) 12 hours prior to therapy.

Serum deprivation is a commonly used method to reduce serum-drug interactions - but also has profound cellular effects including accumulation and synchronization of cells in the G1/G0 [26]. Moreover, given the high protein binding of quizartinib ( $99 \%$ protein bound), it is not surprising that changes in serum concentration would affect drug potency in cell-based models.

For example, we repeated our experiments with the KIT D816V-positive cell line HMC1.2, but this time cultured the cells in serum-reduced media overnight prior to quizartinib treatment the next day. This change in experimental conditions profoundly altered the antiproliferative effect of quizartinib, as the IC50 for serumdeprived cells was $\sim 260 \mathrm{nM}$ compared to the serum replete conditions (IC50 1700 nM). The potency of the serum-deprived cells is in the range of the predicted IC50 for the D816V mutation reported by Zarrinkar et al. [24] (Table 1).

This effect was further validated using an isogenic cell model as well as native FLT3 ITD positive leukemia blasts as described below. (Please, refer to Table 2 and Additional file 1: Figure S1 for similar experiments using native cells and to Table 3 for serumdeprivation experiments using Ba/B3 FLT3 ITD and KIT D816V cells).

\section{Quizartinib induces apoptosis in in vitro leukemia cell lines}

The extraordinary antiproliferative effect seen in some cell models tested, was also accompanied by microscopically condensed pyknotic cells that accumulated over time. This observation suggests that quizartinib may induce apoptosis via inhibition of (mutant) FLT3, KIT or PDGFRA.

Using an annexin V-based immunofluorescence assay, we were able to demonstrate potent dosedependent induction of apoptosis in several leukemia cell lines:

In analogy to the demonstrated antiproliferative effects, evaluation of quizartinib in several cell lines lacking an activated type III RTK (K562, Jurkat and HL60) did not reveal any significant proapoptotic effects. In contrast, cell lines harboring FLT3 ITD (MV4;11, 
Table 1 Non-linear regression analysis of IC50s (Antiproliferation)

\begin{tabular}{|c|c|c|}
\hline \multirow[t]{2}{*}{ Cell line } & \multirow[t]{2}{*}{ Target } & \multirow{2}{*}{$\begin{array}{c}\text { IC50 }(\mathrm{nM}) \\
\text { Inhibition of proliferation }\end{array}$} \\
\hline & & \\
\hline HMC1.1 & KIT V560G & 14 \\
\hline $\mathrm{HMC1} 1.2$ & KIT V560G/D816V & 1727 \\
\hline $\mathrm{HMC} 1.2,0.5 \%$ FBS & KIT V560G/D816V & 263 \\
\hline p815 & KIT D814Y (murine) & 445 \\
\hline Kasumi-1 & KIT N822K & 36 \\
\hline $\mathrm{M}-07 \mathrm{e}+\mathrm{SCF}$ & KIT-activated & 77 \\
\hline M-07e + GM-CSF & GM-CSF signaling & not reached* \\
\hline EOL-1 & FIP1L1-PDGFRA & 1 \\
\hline K562 & $B C R / A B L$ & not reached* \\
\hline HL60 & unknown & not reached* \\
\hline Jurkat & unknown & not reached* \\
\hline MV4;11 & FLT3 ITD (hemizygous) & $<1$ \\
\hline MOLM14 & FLT3 ITD & $<1$ \\
\hline MOLM14 + DMSO & FLT3 ITD & not reached* \\
\hline Pat.221 & CBF AML (KIT WT) & 675 \\
\hline Pat.279 & CBF AML (KIT WT) / FLT3 amplification (subclone)? & 3434 \\
\hline Pat.299 & CBF AML (KIT WT) & 7248 \\
\hline Pat.305 & CBF AML (KIT WT) & 7079 \\
\hline Pat.375 & CBF AML (KIT N/A) & 503 \\
\hline Pat.379 & CBF AML (KIT WT) & 806 \\
\hline Pat.368 & FLT3 amplification? & 2700 \\
\hline Pat.601 & FLT3 ITD & 1153 \\
\hline Pat.176 & FLT3 ITD (Beta1) & not reached* \\
\hline Pat.602 & FLT3 ITD (Beta1) & not reached* \\
\hline
\end{tabular}

* tested up to $10000 \mathrm{nM}$.

The table summarizes estimated IC50 values obtained by non-linear regression analysis for the antiproliferative activity of quizartinib in leukemia cell lines and primary native leukemia blasts.

The cell line HMC1.2 was additionally pre-treated with reduced serum (0.5\% FBS) to address the influence of methodology aspects on sensitivity profiles. To exclude solvent-associated non-specific cytotoxicity, the MOLM-14 cell line was treated with DMSO using the highest concentration for the quizartinib dose experiments.

MOLM14), FIP1L1-PDGFRA (EOL-1), SCF (but not GM-CSF)-stimulated wild-type KIT (M0-7e), or certain KIT mutations (KIT exon 11 V560G, HMC1.1; Kasumi KIT exon $17 \mathrm{~N} 822 \mathrm{~K}$ ) potently underwent apoptosis upon quizartinib exposure with IC50s in the lower nanomolar ranges (Table 2). Notably, IC50s were similar or somewhat higher compared to the antiproliferative effects achieved in these cell lines (compare with Table 1).

HMC1.2, the sister cell line of HMC1.1 harboring an additional KIT D816V mutation, revealed a complete loss of sensitivity towards quizartinib in all tested doses (Figure 2; IC50s are provided with Table 2). This finding suggests that the distinct mutant-KIT isoform directly orchestrates sensitivity towards quizartinib. In this context it is noteworthy, that the KIT D814Y-positive (D816Y in human KIT) murine cell line p815 was still capable to induce apoptosis with an IC50 in the hundreds nanomolar range (Figure 2).

\section{Comparison of quizartinib sensitivity towards different leukemia-driving KIT and FLT3 mutations in an isogenic cellular background}

Quizartinib potently inhibits cellular proliferation and induces apoptosis in leukemia cell lines that are dependent on FLT3, KIT or PDGFRA activity. However, the potency of quizartinib differs widely between the tested cell lines - from complete insensitivity to doses in the low nanomolar range.

The divergent inhibitory effects may be due to differential sensitivity profiles of different (mutant) RTK isoforms (compare findings about HMC1.1 versus HMC1.2 
Table 2 Non-linear regression analysis of IC50s (Apoptosis/Viability)

\begin{tabular}{|c|c|c|}
\hline Cell line & Target & $\begin{array}{c}\text { IC50 (nM) } \\
\text { Induction of apoptosis }\end{array}$ \\
\hline $\mathrm{HMC1} 1$ & KIT V560G & 31 \\
\hline $\mathrm{HMC1} 1.2$ & KIT V560G/D816V & not reached* \\
\hline p815 & KIT D814Y (murine) & 341 \\
\hline Kasumi-1 & KIT N822K & 67 \\
\hline $\mathrm{M}-07 \mathrm{e}+\mathrm{SCF}$ & KIT-activated & 78 \\
\hline M-07e + GM-CSF & unspecific stimulation & not reached* \\
\hline EOL-1 & FIP1L1-PDGFRA & $<1$ \\
\hline K562 & $B C R / A B L$ & not reached* \\
\hline HL60 & N/A & not reached* \\
\hline Jurkat & N/A & not reached* \\
\hline MV4;11 & FLT3 ITD (hemizygous) & 2 \\
\hline MOLM14 & FLT3 ITD & 3 \\
\hline GIST822 & KIT K642E & 109 \\
\hline GIST48 & KIT V560D/D820A & not reached* \\
\hline Pat.368 & FLT3 amplification? & 2998 \\
\hline Pat.601 & FLT3 ITD & 876 \\
\hline Pat.695, 20\% FBS & FLT3 ITD & 2335 \\
\hline Pat.695, 0.5\% FBS & FLT3 ITD & 25 \\
\hline Pat.139, 20\% FBS & FLT3 ITD, Relaps & 760 \\
\hline \multirow[t]{2}{*}{ Pat.139, 0.5\% FBS } & FLT3 ITD, Relaps & 10 \\
\hline & & Reduction of viable cells \\
\hline Pat.507 & CBF AML (KIT WT) & 1275 \\
\hline Pat.317 & CBF AML (KIT D816Y) & 1294 \\
\hline Pat.521 & CBF AML (KIT WT) & 2018 \\
\hline Pat.305 & CBF AML (KIT WT) & 2954 \\
\hline Pat.511 & CBF AML (KIT WT) & 4272 \\
\hline Pat.281 & CBF AML (KIT WT) & 5758 \\
\hline Pat.279 & CBF AML (KIT WT) / FLT3 amplification? (subclone) & 6607 \\
\hline Pat.523 & CBF AML (KIT WT) & 7175 \\
\hline Pat.361 & CBF AML (KIT D816V) & 8443 \\
\hline Pat.239 & CBF AML (KIT D816V) & not reached* \\
\hline
\end{tabular}

* tested up to $10000 \mathrm{nM}$.

The table summarizes estimated IC50 values obtained by non-linear regression analysis for the cytotoxic activity of quizartinib in leukemia cell lines and primary native leukemia blasts. Native patient blasts were cultured in $20 \%$ FBS." to "Cell lines were cultured in $10 \%$ FBS; native patient blasts were cultured in $20 \%$ FBS. To address methodology aspects towards sensitivity profiles two native FLT3 ITD + patient samples (Pat. 695 with newly diagnosed AML, Pat. 139 with relapsed AML) were co-treated with reduced serum (0.5\% FBS).

In addition IC50 estimates for the proapoptotic effect of quizartinib in the imatinib-sensitive GIST solid tumor cell line GIST882, harboring a K642E mutation, and the imatinib-insensitive cell line GIST48, harboring a V560D mutation in addition to a D820A mutation in the tyrosine kinase domain, are provided, revealing sensitivity profiles similar to imatinib.

cells) - but may also have been obscured by additional genomic abnormalities contributing to leukemogenesis and resistance to therapeutics.

To exclude cell line-specific off-target biology interfering with the effects of kinase-inhibition, we tested leukemia-driving RTK mutations in an isogenic cellular background: Various human (mutant) FLT3 or KIT isoforms were stably transfected in the IL3-dependent murine pro B-cell line $\mathrm{Ba} / \mathrm{F} 3$. Activation of the transfected mutant isoforms was demonstrated by selecting for cells with IL-3 growth factor-independent proliferation. However, BaF3 cells expressing wildtype KIT or FLT3 isoforms required the addition of the corresponding ligand, (KIT (SCF) or FLT3 (FLT3L)).

We were able to directly cross-check the clinically most relevant RTK mutations in acute leukemia (i.e. 
Table 3 Estimated IC50s for the proapoptotic and antiproliferative effects of quizartinib in an isogenic cell model of $\mathrm{Ba} / \mathrm{F3}$ cells transfected with various mutant TKs

\begin{tabular}{|c|c|c|c|c|c|}
\hline \multirow[t]{3}{*}{ Isoform } & \multirow[t]{3}{*}{ Mutation locus } & IC50 (nM) & IC50 (nM) & IC50 (nM) & IC50 (nM) \\
\hline & & \multicolumn{2}{|c|}{$\mathrm{Ba} / \mathrm{F} 3$ transfectants } & \multicolumn{2}{|c|}{ leukemia cell lines } \\
\hline & & Inhibition of proliferation & Induction of apotosis & Inhibition of proliferation & Induction of apotosis \\
\hline$B C R / A B L$ & fusion & n.r..$^{*}$ & n.r.* & not reached (K562) & n.r.* (K562) \\
\hline FLT3 WT & $\mathrm{N} / \mathrm{A}$ & 49 & 11 & & \\
\hline FLT3 ITD, 10\% FBS & juxtamembrane domain & 9 & 5 & $<1$ (MOLM14) & 3 (MOLM14) \\
\hline FLT3 ITD, 0.5\%FBS & juxtamembrane domain & $<1$ & N/D & & \\
\hline FLT3 K663Q & tyrosine kinase domain I & 14 & 23 & & \\
\hline FLT D835V & tyrosine kinase domain II & 172 & 888 & & \\
\hline FLT3 D835Y & tyrosine kinase domain II & 84 & 24 & & \\
\hline KIT WT & N/A & 474 & n.r. ${ }^{*}$ & 77 (MO-7e) & 210 (MO-7e) \\
\hline KIT D816F & tyrosine kinase domain II & 2871 & 6254 & & \\
\hline KIT D816V, 10\% FBS & tyrosine kinase domain ॥ & 3074 & 8982 & 1727 (HMC1.2) & n.r.* (HMC1.2) \\
\hline KIT D816V, 0.5\% FBS & tyrosine kinase domain II & 633 & N/A & & \\
\hline KIT D816Y, 0.5\% FBS & tyrosine kinase domain II & 366 & 611 & $445(p 815)$ & $341(p 815)$ \\
\hline parental & N/A & n.r. ${ }^{*}$ & n.r. ${ }^{*}$ & & \\
\hline parental + DMSO & N/A & n.r. ${ }^{*}$ & n.r. ${ }^{*}$ & & \\
\hline
\end{tabular}

*(not reached with tested doses up to $10000 \mathrm{nM}$ ).

Sensitivity of quizartinib is distinct to and differs widely in between different tyrosine kinase isoforms transfected into an isogenic Ba/F3 cellular background. Estimated IC50s were computed using non-linear regression analysis of an average mean of at least 3 experiments for each cell line.

If applicable, IC50s of leukemic cell lines harboring a similar mutation are provided (rows on the right).

Influence of serum-deprivation on sensitivity profiles of quizartinib was tested in two cell strains (Ba/F3 FLT3 ITD or KIT D816V): Cells were cultured in media with a reduced serum concentration $(0.5 \% \mathrm{FBS})$ and treated with quizartinib the next day.

Solvent-associated non-specific cytotoxicity was excluded using the parental Ba/F3 cell strain treated with DMSO in the highest concentration used for the quizartinib dose experiments.

FLT3-ITD, KIT D816V/Y, BCR/ABL1) transfected into an isogenic $\mathrm{Ba} / \mathrm{F} 3$ background against a panel of leukemic cell lines harboring a corresponding RTK mutation. Comparison of inhibition of cellular proliferation after quizartinib treatment revealed strong correlation between naturally occurring and engineered cell lines expressing identical mutant kinases (Figure 3A): Ba/F3 cells stably transfected with a vector encoding for a FLT3 ITD were equally highly sensitive to quizartinib compared to the human FLT3 ITD positive leukemia cell line MOLM14. Vice versa, transfection of a KIT D816V mutation retained $\mathrm{Ba} / \mathrm{F} 3$ cells highly resistant towards quizartinib, which is consistent with findings in the human mast cell leukemia cell line HMC1.2 as discussed earlier. Interestingly, replacing the valine substitution with tyrosine at codon 816 (D816Y) rendered Ba/F3 cells to relative sensitivity to quizartinib - so was the KIT D814Y-positive cell line p815.

This observation is not unique to quizartinib - but is in line with previous data for other KIT tyrosine kinase inhibitors, such as dasatinib [27]. In this context, a recent study suggested structural reasons that underlay drug sensitivity of different mutant-KIT kinases using sunitinib and imatinib mesylate [28].
Surprisingly, transfection of $B C R / A B L 1$ into $\mathrm{Ba} / \mathrm{F} 3$ cells not only did not halt proliferation of cells - but did confer a proliferation advantage for quizartinib treated cells in a dose-dependent manner. This observation deserves further exploration with regard to molecular mechanisms.

Together, these findings suggest a direct mutantspecific tyrosine kinase-mediated effect of quizartinib towards modulation of cellular proliferation. Table 3 provides additional information of sensitivity patterns, with regard to inhibition of proliferation as well as induction of apoptosis, for several mutant-FLT3, -KIT and BCR/ $A B L 1$ isoforms transfected into an isogenic $\mathrm{Ba} / \mathrm{F} 3$ cellular background: Of note, transfection of a FLT3 D835V kinase domain mutation, which is homologous to $\mathrm{D} 816 \mathrm{~V}$ in KIT, reveals restricted sensitivity towards quizartinib - which is in line with a recent study by Smith and colleagues demonstrating a conformational clash preventing proper binding of quizartinib to the FLT3 binding pocket [29]. Importantly, our data further show that alternative substitution of aspartic acid with a tyrosine residue (D835Y) renders cells to sensitivity, which underlines our findings for KIT D816Y as discussed above. 


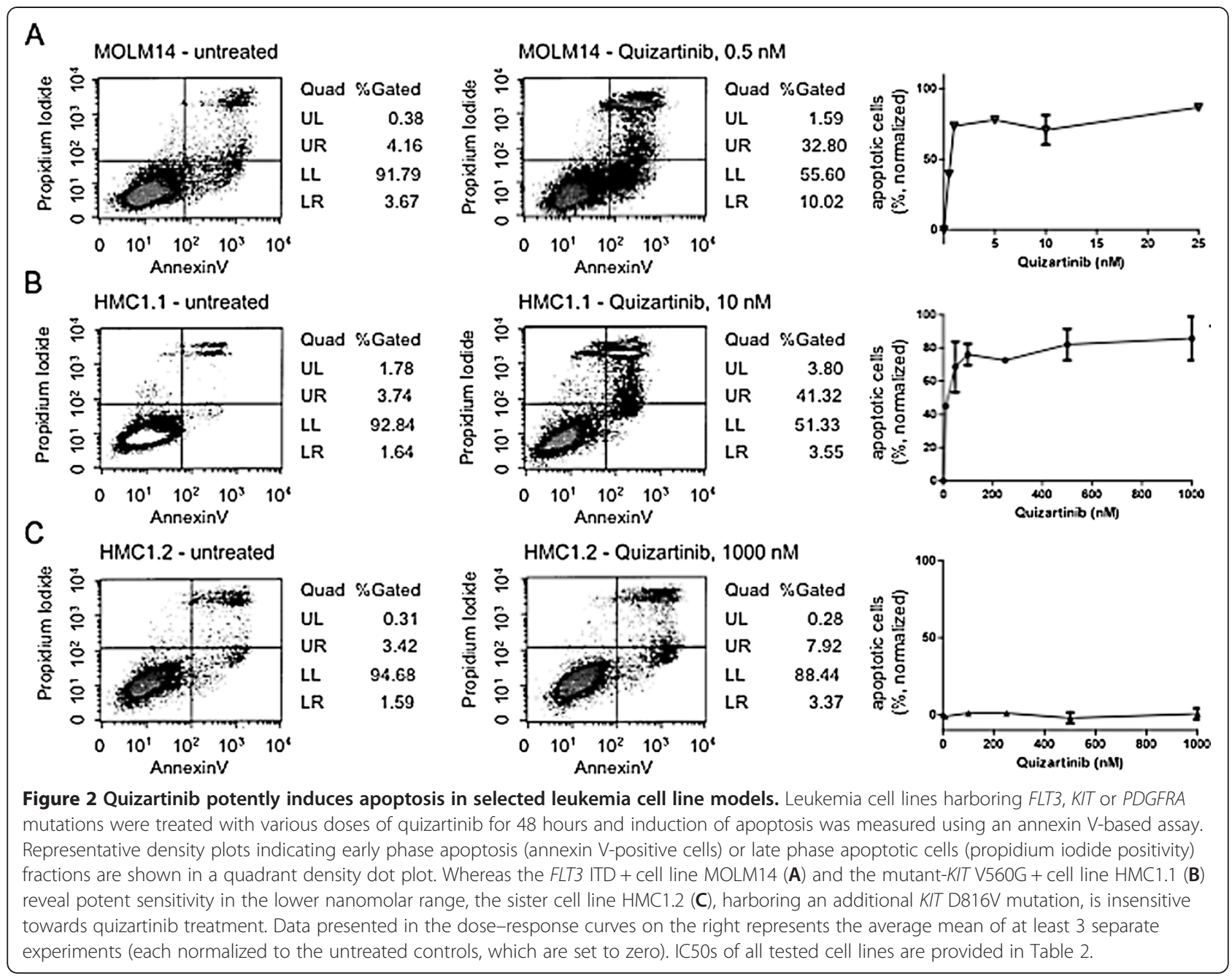

Inhibition of cellular proliferation associates with distinct inhibition of phosphorylation of the target receptor tyrosine kinase in an isogenic cell model

In our cell biology experiments, the sensitivity of the tested cell lines to quizartinib was linked to inhibition of (mutant) class III RTK. The isogenic cell models confirm IC50s obtained for the leukemia cell lines harboring similar mutations, further suggesting a direct interaction of tyrosine kinase inhibition and the observed antiproliferative and proapoptotic effects in the tested cell lines (rather than off-target effects).

To address this question at the protein level, we additionally performed immunoblotting experiments for $\mathrm{Ba} /$ F3 cell lines transfected with mutant KIT or FLT3 kinases and treated with quizartinib for 90 minutes. Indeed, sensitivity towards quizartinib, as indicated by loss of RTK autophosphorylation, proved to be kinasespecific and was in agreement with the functional assays using the same cell lines. Notably, the KIT D816V mutation, exchanging aspartic acid for valine at codon 816 and thus rendering the kinase autophosphorylated, does not show significant reduction of phosphorylation levels - while tyrosine or phenylalanine substitutions at the same codon (D816Y or D816F), similarly leading to autoactivity of the kinase, proof to be sensitive towards quizartinib treatment with loss of autophosphorylation in the nanomolar ranges (Figure 3B). Interestingly, D816Y was thereby dephosphorylated at the glycosylated membrane-bound ( 145 KDa), as well as the intracellular isoform $(\sim 125 \mathrm{KDa})$ - whereas sensitivity of D816F was virtually restricted to the glycosylated isoform.

These results are in line with the viability assays provided in Figure 3A and Table 3, and again argue against nonspecific off-target - but for TK-mediated effects. Moreover, it underlines that TKI sensitivity patterns are not just tyrosine kinase, kinase-domain or codon specific - but may even depend on the type of amino acid substitution at a given codon. 


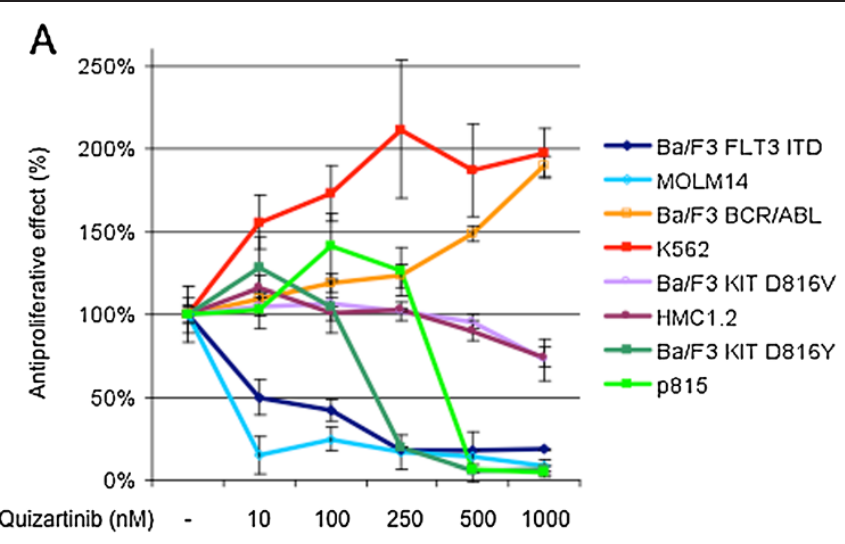

B

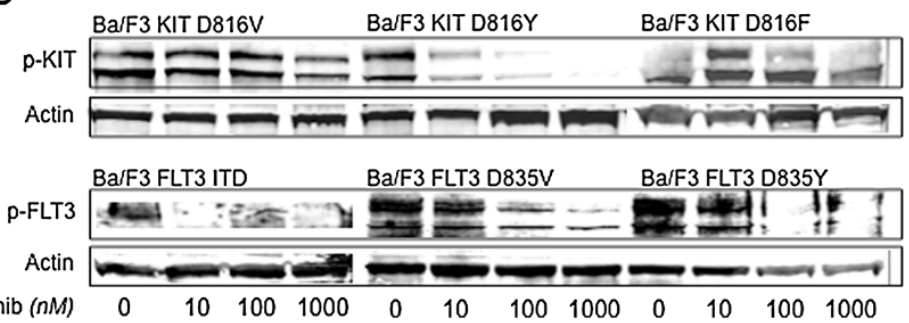

Figure 3 Cellular effects of quizartinib are tyrosine kinase-mediated (A) Quizartinib displays distinct antiproliferative effects of genetically altered $\mathrm{Ba} / \mathrm{F3}$ cells in dependence of the tyrosine kinase isoform transfected. The sensitivity of inhibition of proliferation is thereby similar to the sensitivity achieved in natural leukemia cell lines harboring a similar mutation. Estimated IC50s are provided in Table 3 along with IC50s for the proapoptotic effects in the same cellular context. (B) The observed cellular effects are directly linked to the potency of inhibition of phosphorylation of mutant-KIT and FLT3 isoforms. Whole cell lysates of Ba/F3 cells transfected with different human mutant-KIT or FLT3 isoforms were immunoblotted using a pan-phosphotyrosine antibody or a total-KIT or -FLT3 antibody. Pretreatment of cells with quizartinib revealed isoform-specific inhibition of phosphorylation. Notably, inhibition of phosphorylation of the D816V mutation was significantly reduced compared to the D816Y and D816F isoforms.

In vitro inhibition of cellular proliferation by quizartinib translates into ex vivo antiproliferative effects in native leukemia blasts

We further evaluated the antiproliferative effects of quizartinib using native blasts isolated from patients with newly diagnosed FLT3- or KIT-activated AML (additional patient characteristics are provided in Additional file 2: Table S1 with the online version of the article).

Notably, quizartinib was able to inhibit proliferation of ex vivo CBF AML blasts and FLT3 ITD positive blasts in the upper nanomolar or lower micromolar ranges (Table 1).

CBF AML is associated with high CD117 (i.e. KIT) expression and/or autoactivating mutations within the KIT gene [7]. KIT mutation screening of exons 8, 9, 11, 13 and 17 was performed. No autoactivating mutation in our patient cohort used for antiproliferation assays was detected, suggesting a paracrine activation of KIT in the quizartinib-responsive patient cohort as demonstrated earlier for $\sim 50 \%$ of FLT3/KIT wildtype patients [30].

In FLT3-associated leukemia patients, the antiproliferative effect of quizartinib was inconsistent - with refractory as well as sensitive cases identified. For example, one case sensitive to quizartinib treatment was from a patient with an MLLT3-MLL rearrangement (patient \#368). We were not able to detect any FLT3 or KIT mutations in this patient - although, karyotyping revealed trisomy of chromosome 13 (FLT3 genomic location), which potentially contributed to treatment response via FLT3 amplification. Potent inhibition of amplified FLT3 wildtype gene via quizartinib was recently shown in an in vitro leukemia cell model using the SEM-K2 ALL cell line by Gunawardane and colleagues [31]. Another of our cases demonstrating sensitivity towards quizartinib harbored a FLT3 ITD, but interestingly, two more cases with a FLT3 ITD were refractory to quizartinib (patient \#176 and \#602).

FLT3 ITD sequencing revealed that the internal tandem duplication was located in the beta1 sheet of the first tyrosine kinase domain in both resistant cases. This particular class of mutant kinase is resistant to FLT3 inhibition by midostaurin (PKC412) and is associated with a poor clinical outcome [32-34]. Our data suggests a similar sensitivity profile for quizartinib against FLT3 ITD-beta1 mutant kinases. 


\section{Quizartinib induces apoptosis in ex vivo native leukemia cells}

We next tested isolated native blasts derived from patients with newly diagnosed AML to confirm the proapoptotic effect observed for quizartinib in in vitro leukemia and isogenic mutant-TK models (IC50s for all patients are provided with Table 2; patient characteristics are available as supplementary material in Additional file 2: Table S1).

One sample, taken from a bone marrow aspirate of a patient with de novo AML, was identified to harbor a FLT3 ITD mutation in the juxtamembrane domain of the gene (patient \#601). IC50 was in the higher nanomolar range - which is considerably higher compared to the in vitro FLT3 ITD models. The cause of this discrepancy is unknown, but is commonly observed in ex vivo blasts compared to in vitro models [35,36]. In addition to the above comments about the effect of serum concentration on sensitivity to quizartinib, other genomic abnormalities acquired in the context of complex cytogenetic AML may have contributed to the observed effects in cultured ex vivo blasts.

In a second patient sample, obtained from the bone marrow aspirate of an elderly patient with $M L L$-associated AML - including trisomy of chromosome 13 (leading to overrepresentation of the FLT3 gene), quizartinib treatment induced apoptosis in these cells with an IC50 $\sim 3000 \mathrm{nM}$. Due to the high bioavailability of quizartinib, ex vivo IC50s in the lower micromolar ranges may translate into antileukemic activity in vivo - but this observation needs clinical validation.
A subset of native leukemia samples analyzed presented with a high proportion of dead/apoptotic cells in the untreated control samples. This obscured conventional analysis via annexin/PI staining of proapoptotic effects induced by quizartinib treatment. Nevertheless, we were able to assess reduction of the proportion of viable cells in a cohort of CBF AML patients 48 hours after quizartinib treatment compared to treatment-naive cells (Figure 4 for representative analysis of one patient). Table 2 provides a summary of IC50s of all tested samples.

The cytotoxic effect of quizartinib varied widely. Analysis of the KIT mutation status revealed a wildtype gene for $K I T$ in most patients - some of these were sensitive to quizartinib therapy, arguing for paracrine activation of KIT or FLT3 [30].

Of interest, similar to our cell line results, two patients with a KIT D816V mutation revealed relative insensitivity towards quizartinib. However, as predicted by our in vitro models (see also Figure 3 ), one patient with $\mathrm{CBF}$ AML harboring the less common KIT D816Y mutation demonstrated sensitivity with an IC50 for reduction of viable cells of approximately $1300 \mathrm{nM}$. Notably, this lies in the same range as seen for the proapoptotic effect of ex vivo blasts of a newly diagnosed treatment-naive patient (patient \#601) harboring a FLT3 ITD (JM) mutation. Additional patient characteristics are provided in Additional file 2: Table S1 with the online version of the article.

In general, the reported IC50s in our study for native leukemia cells lie in contrast to a previous report by

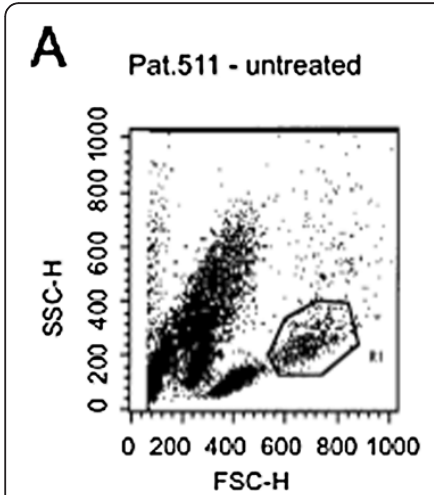

R1, $n=1217$

counted events 15285

viable cells (normalised): 1
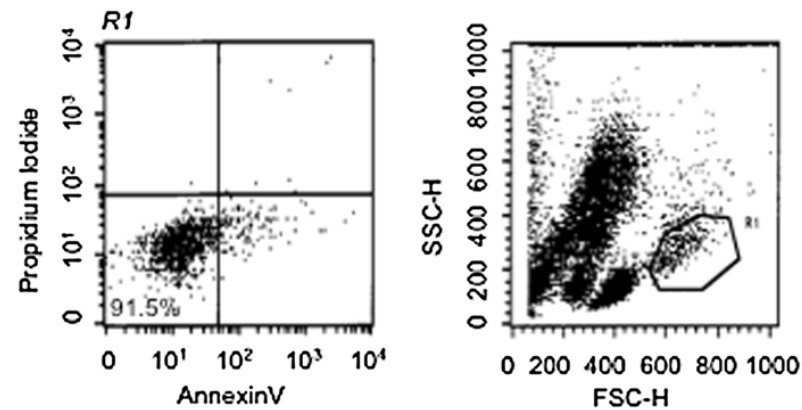

R1, $n=768$

counted events 15000

viable cells (normalised): 0.68

Figure 4 Quizartinib reduces the proportion of viable cells in samples of native leukemia cells. Patient samples were cultured in DMEM containing 20\% FBS and treated with different concentrations of quizartinib for 48 hours. (A) The plot of one patient is shown. The mononuclear cell fraction was gated in a FSC/SSC scatter and viability was confirmed using an annexin V/propidium iodide (PI) stain demonstrating negativity for annexin $\mathrm{V}$ and PI. (B) Relative reduction of the proportion of viable cells in response to quizartinib was assessed by flow cytometry. A doseeffect plot of all tested concentrations of quizartinib is shown on the right. Estimated IC50s by non-linear regression analysis of all tested patient samples are provided in Table 2. 
Zarrinkar and colleagues [24], which have suggested IC50s for FLT3 ITD-positive native blasts in the lower nanomolar ranges.

Several issues need to be discussed in this context. Besides individual cell-context specific additional effects (such as additional mutations rendering signal transduction pathways or drug sensitivities), which may have obscured TK-targeted effects of quizartinib, a couple of methodology-related aspects need to be addressed: As discussed earlier, we did not use serum reduced conditions in our assays - but serum-rich media containing $10 \%$ FBS for cell lines and 20\% FBS for experiments involving native blasts. Moreover, it has been reported that blasts obtained from patients with relapsed FLT3 ITD-positive leukemia may show higher sensitivities towards tyrosine kinase inhibitors due to a higher addiction to FLT3 gain-of-function signal transduction of leukemia blasts in the relapse setting compared to de novo AML samples [37]. Notably, the Zarrinkar study evaluated the efficacy towards quizartinib in relapsed patients while our work included only specimens from newly diagnosed patients. We addressed these issues and treated a newly diagnosed patient with AML as well as a patient with relapsed AML, both harboring a FLT3 ITD mutation, with quizartinib in a dosedependent manner. Both samples were cultured in serum-repleted (20\% FBS) as well as serum-reduced $(0.5 \%$ FBS $)$ conditions. In line with our theory, the average concentration to induce apoptosis was markedly reduced with IC50s in the low nanomolar range in samples cultured in serum-reduced conditions. Even more, sensitivity towards quizartinib was increased in the relapsed leukemia patient. Exemplary AnnexinVbased density plots illustrating the influence of culture conditions with regard to the achievable proapoptotic effects $(\sim 22 \%$ versus $80 \%$ dead/apoptotic cells upon exposure to $10 \mathrm{nM}$ quizartinib when cultured in $20 \%$, resp. $0.5 \%$ FBS) are provided as Additional file 1: Figure S1; IC50s are provided with Table 2.

\section{Antitumor activity of quizartinib in mutant-KIT solid tumor cell lines}

Besides acute leukemia, KIT mutations are found in a large proportion of gastrointestinal stromal tumors (GIST) [38], in subsets of seminomas [39] and melanoma [40]. PDGFR mutations are further reported in myeloproliferative disorders and GIST as well [38].

KIT or PDGFRA tyrosine kinase inhibition is the only known medical treatment option for advanced GIST. Due to the excellent bioavailability properties of quizartinib, higher plasma concentrations are achieved compared to other inhibitors with a similar sensitivity profile. This may be advantageous in particular for the treatment of solid tumor lesions.
We treated an imatinib-sensitive GIST cell line (GIST822) harboring a KIT exon 13 mutation (K642E) and a second cell line, GIST48, harboring an imatinibsensitive V560D mutation plus a secondary imatinibinsensitive activation loop mutation (D820A) with varying concentrations of quizartinib. Due to a much slower in vitro cell doubling time of the GIST cell lines compared to leukemic cell lines, GIST cells were treated for 7 days. Figure 5 demonstrates a potent proapoptotic effect of quizartinib targeting the KIT K642E mutation in the GIST822 cell line, whereas the imatinib-insensitive cell line GIST48 did not display any significant signs of induction of apoptosis following quizartinib treatment. Calculated IC50s are provided in Table 2.

\section{Discussion}

Tyrosine kinase inhibitors are rapidly entering into the clinic. These agents are subject of intensive clinical investigation for the treatment of acute leukemia. For example the following inhibitors are under investigation for mutant $A B L 1, F L T 3$ or KIT-associated subtypes (clinicaltrials.gov): sorafenib (e.g. AML: NCT00217646, NCT00373373), sunitinib (e.g. AML: NCT00783653), dasatinib (e.g. CBF-AML: NCT00850382; SM: NCT00979160; Ph + ALL: NCT00103701, NCT00940524), imatinib (e.g. AML: NCT00707408, NCT00744081; activated RTKs/ various tumors: NCT00171912), lestaurtinib (e.g. AML: NCT00030186, NCT00079482), tandutinib (e.g. AML: NCT00064584, NCT00274248), masitinib (e.g. SM: NCT00814073) and nilotinib (e.g. AML: NCT01222143; SM/HES: NCT00109707).

Quizartinib (formerly AC220), a N-(5-tert-butylisoxazol-3-yl)-N'-\{4-[7-(2-morpholin-4-yl-ethoxy)imidazo [2,1-b] [1,3] benzothiazol 2-yl]phenyl\}urea dihydrochloride, is a novel class III tyrosine kinase inhibitor with promising in vitro as well as in vivo activity against FLT3 wildtype and mutant isoforms [24]. Compared to other tyrosine kinase inhibitors in evaluation for the treatment of acute leukemia subtypes, quizartinib provides superior bioavailability with longer and higher plasma concentrations achieved in vivo [25] thereby targeting and suppressing the activated kinases more effectively.

Early data from a phase II trial of quizartinib in refractory or relapsed FLT3 ITD + patients revealed an acceptable safety profile. Interim analysis of 62 patients (29 female, 33 male) was previously presented (Cortes, EHA annual meeting 2011, \#1019): Common drug-related adverse events for evaluable patients were nausea, QTc prolongation, vomiting, fatigue, dysgeusia, anorexia, febrile neutropenia, diarrhea, and dyspepsia. Drug-related severe adverse events in $>15 \%$ of patients were febrile neutropenia and asymptomatic Grade 3 QTc prolongation. $85 \%$ of patients were evaluable for efficacy: The 

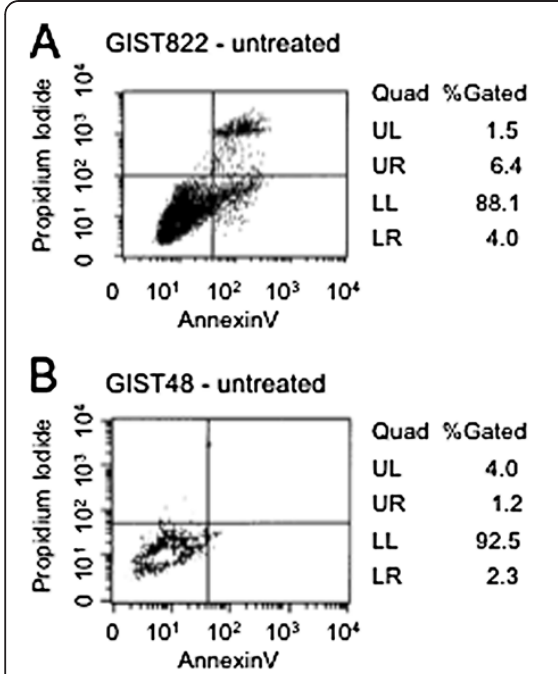
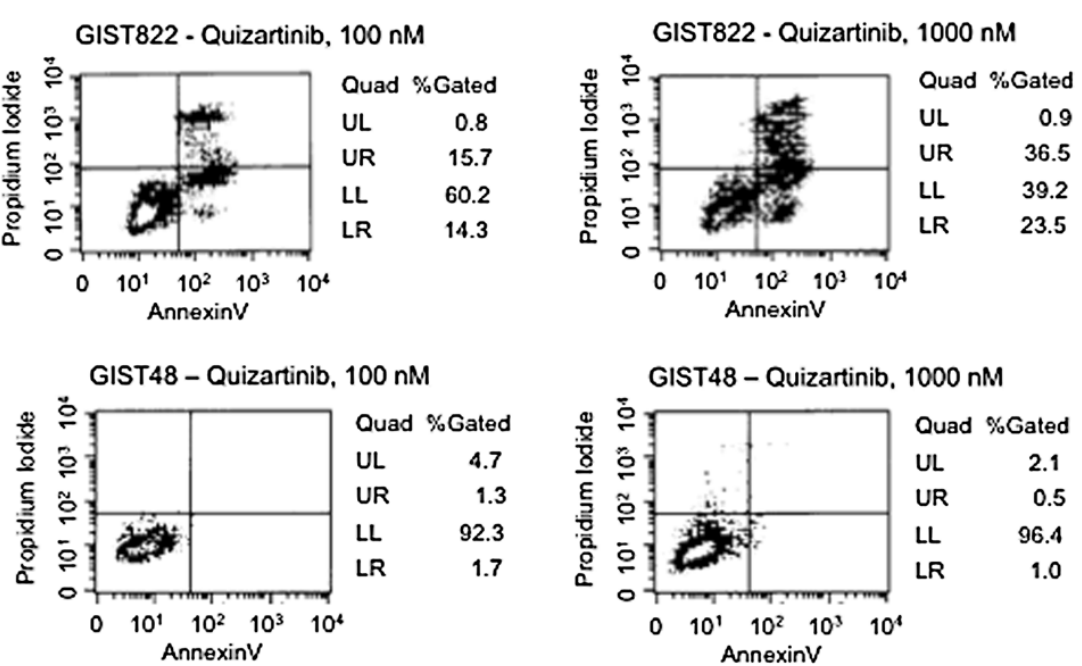

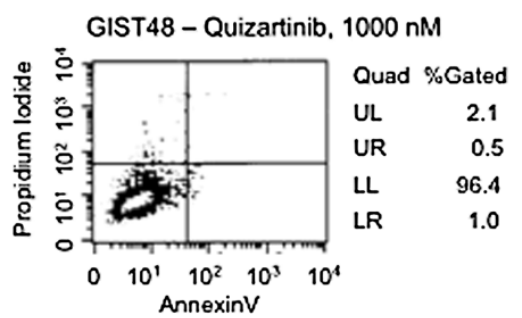

Figure 5 Quizartinib selectively induces apoptosis in gastrointestinal stromal tumor (GIST) cells. The imatinib-sensitive cell line GIST822, harboring a KIT K642E mutation (A) and the imatinib-insensitive cell line GIST48, harboring a KIT V560D plus an additional D820A mutation, were treated with quizartinib for 7 days and assayed for induction of apoptosis. Quadrant density dot plots are provided showing the apoptotic cell fraction as indicated by annexin $V$ positivity (LR quadrant: early apoptosis) and/or positive propidium iodide stain (UR: late apoptosis, UL: dead cells) of quizartinib-treated versus treatment-naive cells. Estimated IC50s by non-linear dose regression analysis are provided with Table 2.

composite $\mathrm{CR}(\mathrm{CRc}=\mathrm{CR}+\mathrm{CRp}+\mathrm{CRi})$ rate was $43 \%(1$ CRp, $22 \mathrm{CRi}$ ) and PR rate was $28 \%$. Of note, of the patients refractory to any prior therapy, 56\% had CRc and $22 \%$ had PR in response to quizartinib treatment. $26 \%$ of patients were bridged to allogenic transplantation concepts.

We now report that quizartinib not only targets FLT3 wildtype and ITD mutant kinases [24] - but also potently inhibits cellular proliferation and induces apoptosis of cells expressing a broad range of other clinically relevant class III (mutant) RTK isoforms associated with various diseases, including pediatric and adult leukemia (FLT3 and KIT), GIST, seminoma and melanoma (KIT), as well as myeloproliferative neoplasms associated with eosinophilia (PDGFRA). Quizartinib-responsive mutations were thereby detected in the juxtamembrane (ITDs), but also the tyrosine kinase domains (TKD I K663Q, TKD II - D835Y) of FLT3, as well as in the KIT JM- (V560G) and TK-domains (D816Y). However, as with all tyrosine kinase inhibitors, quizartinib has a distinct potency profile against different autoactivating mutant RTK isoforms - and some mutations in the TKD of FLT3 (D835V, B1-sheet ITDs) and KIT (D816V) proved to be resistant towards quizartinib. Resistancy of FLT3 ITD mutations located in the beta- 1 sheet of the first TKD has previously been shown for other TKI [32-34] as well. In contrast to the $B C R-A B L 1$ fusion transcript demonstrating resistance towards quizartinib, the FIP1L1-PDGFRA fusion mutation revealed extraordinary sensitivity. In CBF AML, which is frequently dependent upon KIT-mediated (gain-of-function) signal transduction, quizartinib demonstrated varying antiproliferative and cytotoxic efficacy in in vitro and ex vivo leukemia cells - in some cases within the low nanomolar range of FLT3 ITD (JM) positive samples. However, the most prevalent KIT mutation in CBF AML, substituting valine for aspartic acid at codon 816 (KIT D816V), was demonstrated to be basically insensitive towards quizartinib in in vitro and ex vivo leukemia cell lines, primary myeloblasts, and in an isogenic $\mathrm{Ba} / \mathrm{F} 3$ cell model - while substitution of a tyrosine residue (D816Y) retained some sensitivity to quizartinib. Therefore tyrosine kinase genotyping may become a prerequisite for clinical use of this agent.

Moreover, based on our data, we speculate that quizartinib may be a promising agent in solid tumors associated with KIT mutations, such as GIST or melanoma: In addition to a favorable activity against KIT mutant kinases expressed in GIST (and other mutant-KIT neoplasms), the excellent pharmacokinetics with unprecedented achievable plasma concentrations may be advantageous to target bulky solid tumor lesions that have impaired drug uptake. Thus, our data opens new avenues for clinical investigation and further testing of the efficacy of quizartinib in these settings is warranted.

It has to be noted, that IC50s in our studies were coherent in between all cell models used - but higher compared to a previous report [24]. The phenomenon was seen throughout the assays and is therefore most likely due to methodology reasons as we have illustrated with several experimental data:

While individual cell-context specific additional effects (such as additional mutations) can not be fully excluded to have obscured sensitivity profiles in some cell models, 
methodological differences most likely will account for most of the discrepancies observed: In contrast to previous studies using serum-depleted culture conditions $(0.5 \% \mathrm{FBS})$, we used serum-repleted medium in all assays (10\% FBS for cell lines, 20\% for native blasts).

Even more, data from Zarrinkar and colleagues were based on treating refractory/relapsed AML samples [24] - in contrast, samples tested in our assays were isolated from patients with newly diagnosed disease. However, it is believed that refractory/relapsed patient samples have higher sensitivities towards TK-inhibition due to a higher addiction to the respective oncogenic (mutant) tyrosine kinase [37].

To underline our theories, we have provided substantial experimental data using serum-deprived versus serum-rich culture conditions in cell lines and native blasts derived from newly diagnosed as well as relapsed patients to treat with quizartinib in a dose dependent manner. High correlation of serum-levels and achievable IC50s was confirmed in all cell models. In addition, treating newly diagnosed versus relapsed FLT3 ITDpositive leukemia native patient samples, a higher sensitivity profile for relapsed AML was verified. Thus, the data will need to be interpreted in this context.

\section{Conclusion}

To summarize, our findings suggest that quizartinib is a promising agent for treatment of several hematologic and solid human neoplasms. However, due to the quizartinibspecific mutation restricted spectrum of activity, tyrosine kinase mutation screening may be required to identify patients most likely to respond to quizartinib therapy.

\section{Methods}

\section{Cell lines}

The CML blast crisis cell line K562 was a generous gift of Dr. Lopez, Oregon Health and Science University, Portland, OR. The FLT3 ITD positive AML cell line MOLM14 (heterozygous for an ITD of DFREYE at amino acid position 593-598 [2]) was acquired through the Fujisaki Cell Center (Okayama, Japan). The acute myeloid leukemia cell line HL60, the KIT N822K positive CBF AML cell line Kasumi1 [41] and the FLT3 ITD positive AML cell line MV4-11were obtained from the German Collection of Microorganisms and Cell Cultures (DSMZ). MV4-11 cells are hemizygous for an ITD of amino acids VDFREYEYDH at position 592-601 [42]. The interleukin 3 (IL-3)-dependent murine pro-B cell line $\mathrm{Ba} / \mathrm{F} 3$, the FIP1L1-PDGFRA positive cell line EOL-1 [43], from a patient with eosinophilic leukemia following hypereosinophilic syndrome [44], and the p815 mast cell line was obtained from the American Type Culture Collection (ATCC, Manassas, VA). The murine KIT D814Y mutant isoform expressed by the p815 cell line
[45] is homologous to the human KIT D816Y mutation. The human hematopoietic growth factor-dependent M-07e cell line was kindly provided by Dr. Hal Broxmeyer (Department of Microbiology and Immunology, Walther Oncology Center, Indiana University School of Medicine, Indianapolis, IN). The human HMC-1.1 mast cell line, expressing a KIT juxtamembrane domain mutant isoform (V560G), was provided by Dr. Butterfield (Division of Allergic Diseases, Department of Internal Medicine, Mayo Clinic, Rochester, MN). A spontaneously occurring subclone of the HMC-1.1 cell line, HMC-1.2, which has an additional mutation in the activation loop (D816V) [46], was kindly provided by Dr. Akin (Laboratory of Allergic Diseases, National Institute of Allergy and Infectious Diseases, NIH, Bethesda, MD). All cell lines were cultured in RPMI media containing 10\% FBS.

M-07e cells were cultured using recombinant human granulocyte-macrophage colony stimulating factor (GMCSF, R\&D, Minneapolis, MN) as a growth supplement as previously described [47]. Negativity for mycoplasma contamination was confirmed using the pluripotent PCR Mycoplasma test Kit (AppliChem, Darmstadt, Germany). Cell lines harboring a mutant KIT, FLT3 or BCR-ABL1 were sequence confirmed.

The gastrointestinal stromal tumor cell lines GIST822, harboring a KIT exon 13 mutation (K642E), and GIST48, harboring an imatinib-sensitive V560D mutation plus a secondary imatinib-insensitive activation loop mutation (D820A) were generously provided by Dr. Kopp (University of Tübingen) $[48,49]$.

\section{Site-directed mutagenesis and generation of a $\mathrm{Ba} / \mathrm{F} 3$ cell line expressing KIT or FLT3 isoforms}

Site-directed mutagenesis and generation of $\mathrm{Ba} / \mathrm{F} 3$ cell lines stably expressing mutant KIT D816V, D816Y, D816F, FLT3 ITD, D835Y, D835Y, K663Q and FLT3 wildtype were performed as previously described [50-52].

KIT Wildtype cDNA cloned into a pJP1563 plasmid vector was obtained from the DNASU Plasmid Repository at the Biodesign Institute of the Arizona State University (ASU). Lipofection transfection into the parental $\mathrm{Ba} / \mathrm{F} 3$ cell line was performed to stably express KIT Wildtype by double selection for neomycin (pCMVneo plasmid), blasticidin (pJP1563 plasmid) or gentamicin (G418; all other plasmids) resistance and IL-3-independent growth. The Ba/F3 KIT Wildtype cell line was cultured using recombinant human stem cell factor (SCF/KITLigand, R\&D, Minneapolis, MN) as a growth supplement.

\section{Antibodies and reagents}

The small-molecule compounds quizartinib was obtained from Ambit Biosystems and dissolved in DMSO to create $10 \mathrm{mmol} / \mathrm{L}$ stock solutions and stored at $-20^{\circ} \mathrm{C}$. 
Anti-KIT and anti-FLT3 rabbit polyclonal antibodies were used at a 1:5,000 to 1:1,000 dilution. Antiphosphotyrosine p-KIT antibodies (Tyr568/570 and Tyr703), p-FLT3 antibodies (Tyr 589/591) and a pan-antiphosphotyrosine antibody (clone PY20) were administered at dilutions of 1:100 to 1:2,000 (all from Cell Signaling Technology).

Infrared dye-conjugated secondary goat anti-rabbit or anti-mouse antibodies to use in a $\mathrm{LI}-\mathrm{COR}^{\circledR}$ imaging detection system were prepared according to standard protocols (LI-COR Biosciences, Lincoln, NE).

\section{Isolation of bone marrow and peripheral blood mononuclear cells}

Bone marrow aspirate and peripheral blood samples from patients with AML were collected in $5000 \mathrm{U}$ heparin after informed consent and approval of the ethics committee of the University of Tübingen or Ulm. Mononuclear cells were isolated by Ficoll Hypaque density gradient fractionation [35]. Additional acute myeloid leukemia samples were generously provided by the German-Austrian AML Study Group (AMLSG) leukemia biobank (patient characteristics summarized in Additional file 2: Table S1). Native ex vivo blasts were cultured in DMEM media containing 20\% FBS.

\section{Immunoblotting}

Cell pellets were lysed with 100 to $150 \mu \mathrm{L}$ of protein lysis buffer $(50 \mathrm{mmol} / \mathrm{L}$ Tris, $150 \mathrm{mmol} / \mathrm{L} \mathrm{NaCl}, 1 \%$ NP40, 0.25\% deoxycholate with added inhibitors aprotinin, AEBSF, leupeptin, pepstatin, sodium orthovanadate, and sodium pyruvate, respectively phosphatase inhibitor cocktails "2“ and "1" or „3“ (Sigma, St. Louis, MO). Protein from cell lysates (75 to $200 \mu \mathrm{g}$ protein) was used for whole cell protein analysis after denaturing by Western immunoblot assays using a BioRad Criterion system (protein separation by SDS-PAGE in $3-8 \%$ or $10 \%$ polyacrylamide gels followed by electroblotting onto nitrocellulose membranes). Nonspecific binding was blocked by incubating the blots in nonfat dry milk or BSA. Primary antibodies were incubated for one hour or over night, followed by several washes of Tris-buffered saline (TBS) containing $0.005 \%$ Tween 20 . The appropriate secondary antibody was applied for $30^{\prime}$, followed by several washes. Antibody-reactive proteins were detected using a LICOR Odyssey ${ }^{\circledR}$ fluorescence optical system (LI-COR Biosciences, Lincoln, NE).

\section{Apoptosis and cell viability assays}

Induction of apoptosis upon quizartinib treatment was assayed in dilution series $(0-5000 \mathrm{nM})$ and translocation of phosphatidylserine from the inner to the outer leaflet of the plasma membrane as an early indicator of apoptosis was analyzed using an Annexin V-based assay (Immunotech, Marseilles, France) and a FACScalibur ${ }^{\circledR}$ flow cytometer loaded with CellQuest ${ }^{\circledR}$ analysis software (BD, Heidelberg, Germany) [27,47].

A proportion of ex vivo leukemia blasts were not available for induction of apoptosis assays using Annexin V/PI staining due to a higher percentage of apoptotic cells in the untreated negative control population. Nevertheless, viability assays were assessed using FSC/SSC-flow cytometry experiments with a gate on the living cell population. Reduction of viable cells in the presence of quizartinib was measured 48 hours post quizartinib treatment.

\section{Proliferation assays}

Cells were added to 96-well plates at densities of 50000 cells per well. Quizartinib was added in dilution series $(0-5000 \mathrm{nM})$ and proliferation was measured at 48 hours using an 2,3-bis[2-methoxy-4-nitro-5-sulfophenyl]-2Htetrazolium-5-carboxanilide inner salt (XTT)-based assay (Sigma, MO) [27,47].

\section{Polymerase Chain Reaction (PCR) and Sequencing}

Genomic DNA was isolated using a DNeasy ${ }^{\circledR}$ DNA purification kit (Qiagen, Hilden, Germany). FLT3 mutation status was assessed by routine standard PCR techniques. KIT mutation status of exon $8,9,11,13$ and 17 was analyzed by PCR followed by bidirectional sequencing. The primer sets are as follows: KIT exon 8, sense: GCT GAG GTT TTC CAG CAC TC; KIT exon 8, antisense: AAT TGC AGT CCT TCC CCT CT; KIT exon 9, sense: AGCCAGGGCTTTTGTTTTCT; KIT exon 9, antisense: CAGAGCCTAAACATCCCCTTA; KIT exon 11, sense: CCTTTGCTGATTGGTTTCGT; KIT exon 11, antisense: AAACAAAGGAAGCCACTGGA; KIT exon 13, sense: GTTCCTGTATGGTACTGCATGCG; KIT exon 13, antisense: CAGTTTATAATCTAGCATTG CC; KIT exon 17, sense: GGTTTTCTTTTCTCCTCCA ACC; KIT exon 17, antisense: GGATTTACATTATGA AAGTCACAGG.

\section{Data analysis}

Inhibition of proliferation or the proportion of apoptotic/viable cells was assessed in dilution bar diagrams. Non-linear 4-parameter median-effect regression analysis was performed to compute IC50s using Prism ${ }^{\circledR}$ (GraphPad Software, Inc., LaJolla, CA) or MasterPlex ${ }^{\circledR}$ software (Hitachi Solutions, Tokyo, Japan).

\section{Additional files}

Additional file 1: Figure S1. Native FLT3 ITD positive patient blasts were treated with quizartinib in a dose-dependent manner and cultured in serum reduced (0.5\%) versus serum repleted (20\%) conditions. Cells were incubated for 48 hours and induction of apoptosis was measured using an annexin V-based assay. Densitiy plots for quizartinib at $10 \mathrm{nM}$ are shown - estimated IC50s are provided with Table 2. 
Additional file 2: Table S1. Supplementary information on patient characteristics is available at the website of MOLECULAR CANCER (see "Additional file 2: Table S1/Additional file 1: Figure S1").

\section{Abbreviations}

AML: Acute myeloid leukemia; ALL: Acute lymphoid leukemia; CML: Chronic myeloid leukemia; FIP1L1: FIP1-like 1; FLT3: FMS-like tyrosine kinase 3; GIST: Gastrointestinal stromal tumor; HES: Hypereosinophilic syndrome; IC50: Concentration sufficient to achieve a 50\% inhibition; IL3: Interleukin 3: ITD: Internal tandem duplication; KIT: v-kit Hardy-Zuckerman 4 feline sarcoma viral oncogene homolog; PDGFRA: Platelet-derived growth factor receptor alpha; RTK: Class III receptor tyrosine kinases; SM: Systemic mastocytosis; TKD1/2: Tyrosine kinase domain 1 resp. 2; TKI: Tyrosine kinase inhibitor; XTT: 2,3-Bis-(2-methoxy-4-nitro-5-sulfophenyl)-2H-tetrazolium-5-carboxanilidsodium salt.

\section{Competing interests}

The author(s) declare that they have no competing interest.

\section{Authors' contributions}

Dr. KMKS conceived of the design of the study, carried out experiments, analyzed and interpreted data and drafted the manuscript. FA: substantially participated in design and acquisition of experiments, analyzed data and helped drafting the manuscript. Dr. HD conceived of the study, analyzed and interpreted data and critically revised the manuscript. Dr. KD participated in the acquisition of data, analyzed and interpreted data and critically revised manuscript. Dr. MCH designed experiments, analyzed and interpreted data and critically revised manuscript. Dr. MMS conceived of the design of the study, analyzed and interpreted data and drafted the manuscript. All authors read and approved the final manuscript.

\section{Authors' information}

Grant support in part by the Deutsche Krebshilfe Foundation (MMS, KKS), the IZKF Program of the Medical Faculty Tübingen (MMS) and the Carreras Scholarship Program (KKS), a grant from the Leukemia and Lymphoma Society $(\mathrm{MCH})$ and a Merit Review Grant from the Department of Veterans Affairs (MCH). We acknowledge support by Deutsche

Forschungsgemeinschaft and Open Access Publishing Fund of Tübingen University.

\section{Acknowledgements}

We thank Dr. Barbara Illing and Katharina-Henriette Rasp for excellent assistance in data acquisition.

\section{Author details}

'Department of Hematology, Oncology, Rheumatology, Immunology and Pulmology, University Hospital Tübingen, Tübingen, Germany. ${ }^{2}$ Department of Medicine; Division of Hematology and Medical Oncology, Portland VA Medical Center and OHSU Knight Cancer Institute, Portland, OR, USA.

${ }^{3}$ Department of Internal Medicine III, University Hospital UIm, UIm, Germany.

Received: 27 October 2012 Accepted: 19 February 2013 Published: 7 March 2013

\section{References}

1. Nakao M, Yokota S, Iwai T, Kaneko H, Horiike S, Kashima K, Sonoda Y, Fujimoto T, Misawa S: Internal tandem duplication of the flt3 gene found in acute myeloid leukemia. Leukemia 1996, 10:1911-1918.

2. Yokota S, Kiyoi H, Nakao M, Iwai T, Misawa S, Okuda T, Sonoda Y, Abe T, Kahsima K, Matsuo Y, Naoe T: Internal tandem duplication of the FLT3 gene is preferentially seen in acute myeloid leukemia and myelodysplastic syndrome among various hematological malignancies. A study on a large series of patients and cell lines. Leukemia 1997, 11:1605-1609

3. Yamamoto $Y$, Kiyoi H, Nakano $Y$, Suzuki R, Kodera $Y$, Miyawaki S, Asou N, Kuriyama K, Yagasaki F, Shimazaki C, et al: Activating mutation of D835 within the activation loop of FLT3 in human hematologic malignancies. Blood 2001, 97:2434-2439.

4. Ciolli S, Vannucchi AM, Leoni F, Nozzoli C, Longo G, Salati A, Pancrazzi A Bianchi L, Gigli F, Bosi A: Internal tandem duplications of Flt3 gene (Flt3/
ITD) predicts a poor post-remission outcome in adult patients with acute non-promyelocytic leukemia. Leuk Lymphoma 2004, 45:73-78.

5. Taketani T, Taki T, Sugita K, Furuichi Y, Ishii E, Hanada R, Tsuchida M, Ida K, Hayashi Y: FLT3 mutations in the activation loop of tyrosine kinase domain are frequently found in infant ALL with MLL rearrangements and pediatric ALL with hyperdiploidy. Blood 2004, 103:1085-1088.

6. Garcia-Montero AC, Jara-Acevedo M, Teodosio C, Sanchez ML, Nunez R, Prados A, Aldanondo I, Sanchez L, Dominguez M, Botana LM, et al: KIT mutation in mast cells and other bone marrow hematopoietic cell lineages in systemic mast cell disorders: a prospective study of the Spanish Network on Mastocytosis (REMA) in a series of 113 patients. Blood 2006, 108:2366-2372.

7. Beghini A, Peterlongo P, Ripamonti CB, Larizza L, Cairoli R, Morra E, Mecucci C: C-kit mutations in core binding factor leukemias. Blood 2000, 95:726-727.

8. Corbacioglu S, Kilic M, Westhoff MA, Reinhardt D, Fulda S, Debatin KM: Newly identified c-KIT receptor tyrosine kinase ITD in childhood AML induces ligand-independent growth and is responsive to a synergistic effect of imatinib and rapamycin. Blood 2006, 108:3504-3513.

9. Whitman SP, Ruppert AS, Radmacher MD, Mrozek K, Paschka P, Langer C, Baldus CD, Wen J, Racke F, Powell BL, et al: FLT3 D835/I836 mutations are associated with poor disease-free survival and a distinct gene-expression signature among younger adults with de novo cytogenetically normal acute myeloid leukemia lacking FLT3 internal tandem duplications. Blood 2008, 111:1552-1559.

10. Paschka P, Marcucci G, Ruppert AS, Mrozek K, Chen H, Kittles RA Vukosavljevic T, Perrotti D, Vardiman JW, Carroll AJ, et al: Adverse prognostic significance of KIT mutations in adult acute myeloid leukemia with inv(16) and t(8;21): a Cancer and Leukemia Group B Study. J Clin Oncol 2006, 24:3904-3911.

11. Cools J, DeAngelo DJ, Gotlib J, Stover EH, Legare RD, Cortes J, Kutok J, Clark J, Galinsky I, Griffin JD, et al: A tyrosine kinase created by fusion of the PDGFRA and FIP1L1 genes as a therapeutic target of imatinib in idiopathic hypereosinophilic syndrome. N Engl J Med 2003, 348:1201-1214.

12. Pardanani A, Ketterling RP, Brockman SR, Flynn HC, Paternoster SF, Shearer BM, Reeder TL, Li CY, Cross NC, Cools J, et al: CHIC2 deletion, a surrogate for FIP1L1-PDGFRA fusion, occurs in systemic mastocytosis associated with eosinophilia and predicts response to imatinib mesylate therapy. Blood 2003, 102:3093-3096.

13. Zota V, Miron PM, Woda BA, Raza A, Wang SA: Eosinophilia with FIP1L1PDGFRA fusion in a patient with chronic myelomonocytic leukemia. J Clin Oncol 2008, 26:2040-2041.

14. Crump M, Hedley D, Kamel-Reid S, Leber B, Wells R, Brandwein J, Buckstein $\mathrm{R}$, Kassis J, Minden M, Matthews J, et al: A randomized phase I clinical and biologic study of two schedules of sorafenib in patients with myelodysplastic syndrome or acute myeloid leukemia: a NCIC (National Cancer Institute of Canada) Clinical Trials Group Study. Leuk Lymphoma 2010, 51:252-260.

15. Ravandi F, Cortes JE, Jones D, Faderl S, Garcia-Manero G, Konopleva MY, O'Brien S, Estrov Z, Borthakur G, Thomas D, et al: Phase I/II study of combination therapy with sorafenib, idarubicin, and cytarabine in younger patients with acute myeloid leukemia. J Clin Oncol 2010, 28:1856-1862.

16. Fiedler $\mathrm{W}$, Mesters $\mathrm{R}$, Tinnefeld $\mathrm{H}$, Loges $\mathrm{S}$, Staib $\mathrm{P}$, Duhrsen $U$, Flasshove $M$ Ottmann OG, Jung W, Cavalli F, et al: A phase 2 clinical study of SU5416 in patients with refractory acute myeloid leukemia. Blood 2003, 102:2763-2767.

17. Fiedler W, Serve H, Dohner H, Schwittay M, Ottmann OG, O'Farrell AM, Bello $\mathrm{CL}$, Allred R, Manning WC, Cherrington JM, et al: A phase 1 study of SU11248 in the treatment of patients with refractory or resistant acute myeloid leukemia (AML) or not amenable to conventional therapy for the disease. Blood 2005, 105:986-993.

18. Fischer T, Stone RM, Deangelo DJ, Galinsky I, Estey E, Lanza C, Fox E, Ehninger G, Feldman EJ, Schiller GJ, et al: Phase IIB trial of oral Midostaurin (PKC412), the FMS-like tyrosine kinase 3 receptor (FLT3) and multi-targeted kinase inhibitor, in patients with acute myeloid leukemia and high-risk myelodysplastic syndrome with either wildtype or mutated FLT3. J Clin Oncol 2010, 28:4339-4345.

19. Knapper S, Burnett AK, Littlewood T, Kell WJ, Agrawal S, Chopra R, Clark R, Levis MJ, Small D: A phase 2 trial of the FLT3 inhibitor lestaurtinib 
(CEP701) as first-line treatment for older patients with acute myeloid leukemia not considered fit for intensive chemotherapy. Blood 2006, 108:3262-3270.

20. Levis M, Ravandi F, Wang ES, Baer MR, Perl A, Coutre S, Erba H, Stuart RK, Baccarani M, Cripe LD, et al: Results from a randomized trial of salvage chemotherapy followed by lestaurtinib for patients with FLT3 mutant AML in first relapse. Blood 2011, 117:3294-3301.

21. Gotlib J, Berube C, Growney JD, Chen CC, George TI, Williams C, Kajiguchi T, Ruan J, Lilleberg SL, Durocher JA, et al: Activity of the tyrosine kinase inhibitor PKC412 in a patient with mast cell leukemia with the D816V KIT mutation. Blood 2005, 106:2865-2870.

22. Paul C, Sans B, Suarez F, Casassus P, Barete S, Lanternier F, GrandpeixGuyodo C, Dubreuil P, Palmerini F, Mansfield CD, et al: Masitinib for the treatment of systemic and cutaneous mastocytosis with handicap: a phase 2a study. Am J Hematol 2010, 85:921-925.

23. Verstovsek S, Tefferi A, Cortes J, O'Brien S, Garcia-Manero G, Pardanani A, Akin C, Faderl S, Manshouri T, Thomas D, Kantarjian H: Phase II study of dasatinib in Philadelphia chromosome-negative acute and chronic myeloid diseases, including systemic mastocytosis. Clin Cancer Res 2008, 14:3906-3915.

24. Zarrinkar PP, Gunawardane RN, Cramer MD, Gardner MF, Brigham D, Belli B, Karaman MW, Pratz KW, Pallares G, Chao Q, et al: AC220 is a uniquely potent and selective inhibitor of FLT3 for the treatment of acute myeloid leukemia (AML). Blood 2009, 114:2984-2992.

25. Chao Q, Sprankle KG, Grotzfeld RM, Lai AG, Carter TA, Velasco AM, Gunawardane RN, Cramer MD, Gardner MF, James J, et al: Identification of $\mathrm{N}$-(5-tert-butyl-isoxazol-3-yl)-N'-\{4-[7-(2-morpholin-4-yl-ethoxy)imidazo [2,1-b][1,3]benzothiazol-2-yl]phenyl\}urea dihydrochloride (AC220), a uniquely potent, selective, and efficacious FMS-like tyrosine kinase-3 (FLT3) inhibitor. J Med Chem 2009, 52:7808-7816.

26. Mueller S, Schittenhelm M, Honecker F, Malenke E, Lauber K, Wesselborg S, Hartmann JT, Bokemeyer C, Mayer F: Cell-cycle progression and response of germ cell tumors to cisplatin in vitro. Int J Oncol 2006, 29:471-479.

27. Schittenhelm MM, Shiraga S, Schroeder A, Corbin AS, Griffith D, Lee FY, Bokemeyer C, Deininger MW, Druker BJ, Heinrich MC: Dasatinib (BMS354825), a dual SRC/ABL kinase inhibitor, inhibits the kinase activity of wild-type, juxtamembrane, and activation loop mutant KIT isoforms associated with human malignancies. Cancer Res 2006, 66:473-481.

28. Gajiwala KS, Wu JC, Christensen J, Deshmukh GD, DiehI W, DiNitto JP, English JM, Greig MJ, He YA, Jacques SL, et al: KIT kinase mutants show unique mechanisms of drug resistance to imatinib and sunitinib in gastrointestinal stromal tumor patients. Proc Natl Acad Sci USA 2009, 106:1542-1547.

29. Smith CC, Wang Q, Chin CS, Salerno S, Damon LE, Levis MJ, Perl AE, Travers KJ, Wang S, Hunt JP, et al: Validation of ITD mutations in FLT3 as a therapeutic target in human acute myeloid leukaemia. Nature 2012, 485:260-263.

30. Zheng R, Klang K, Gorin NC, Small D: Lack of KIT or FMS internal tandem duplications but co-expression with ligands in AML. Leuk Res 2004, 28:121-126.

31. Gunawardane R, Rooks A, Dao A, et al: Inhibition of FLT3 autophosphorylation and downstream signaling both in vitro and in vivo by AC220, a second generation potent and selective FLT3 inhibitor. Proceedings of the 101st Annual Meeting of the American Association for Cancer Research, 2010 Apr 17-21. Washington, DC. Philadelphia (PA); 2010. Abstract 3619.

32. Breitenbuecher F, Markova B, Kasper S, Carius B, Stauder T, Bohmer FD, Masson K, Ronnstrand L, Huber C, Kindler T, Fischer T: A novel molecular mechanism of primary resistance to FLT3-kinase inhibitors in AML. Blood 2009, 113:4063-4073.

33. Breitenbuecher F, Schnittger S, Grundler R, Markova B, Carius B, Brecht A, Duyster J, Haferlach T, Huber C, Fischer T: Identification of a novel type of ITD mutations located in nonjuxtamembrane domains of the FLT3 tyrosine kinase receptor. Blood 2009, 113:4074-4077.

34. Kayser S, Schlenk RF, Londono MC, Breitenbuecher F, Wittke K, Du J, Groner S, Spath D, Krauter J, Ganser A, et al: Insertion of FLT3 internal tandem duplication in the tyrosine kinase domain-1 is associated with resistance to chemotherapy and inferior outcome. Blood 2009, 114:2386-2392.

35. Schittenhelm MM, Kampa KM, Yee KW, Heinrich MC: The FLT3 inhibitor tandutinib (formerly MLN518) has sequence-independent synergistic effects with cytarabine and daunorubicin. Cell Cycle 2009, 8:2621-2630.

36. Yee KW, Schittenhelm M, O'Farrell AM, Town AR, McGreevey L, Bainbridge T, Cherrington JM, Heinrich MC: Synergistic effect of SU11248 with cytarabine or daunorubicin on FLT3 ITD-positive leukemic cells. Blood 2004, 104:4202-4209.

37. Pratz KW, Sato T, Murphy KM, Stine A, Rajkhowa T, Levis M: FLT3-mutant allelic burden and clinical status are predictive of response to FLT3 inhibitors in AML. Blood 2010, 115:1425-1432.

38. Corless CL, Fletcher JA, Heinrich MC: Biology of gastrointestinal stromal tumors. J Clin Oncol 2004, 22:3813-3825.

39. Kemmer K, Corless CL, Fletcher JA, McGreevey L, Haley A, Griffith D, Cummings OW, Wait C, Town A, Heinrich MC: KIT mutations are common in testicular seminomas. Am J Pathol 2004, 164:305-313.

40. Beadling C, Jacobson-Dunlop E, Hodi FS, Le C, Warrick A, Patterson J, Town A, Harlow A, Cruz F 3rd, Azar S, et al: KIT gene mutations and copy number in melanoma subtypes. Clin Cancer Res 2008, 14:6821-6828.

41. Beghini A, Magnani I, Ripamonti CB, Larizza L: Amplification of a novel CKit activating mutation Asn(822)-Lys in the Kasumi-1 cell line: a t(8;21)Kit mutant model for acute myeloid leukemia. Hematol J 2002, 3:157-163.

42. Quentmeier H, Reinhardt J, Zaborski M, Drexler HG: FLT3 mutations in acute myeloid leukemia cell lines. Leukemia 2003, 17:120-124.

43. Cools J, Quentmeier H, Huntly BJ, Marynen P, Griffin JD, Drexler HG, Gilliland DG: The EOL-1 cell line as an in vitro model for the study of FIP1L1PDGFRA-positive chronic eosinophilic leukemia. Blood 2004, 103:2802-2805

44. Saito H, Bourinbaiar A, Ginsburg M, Minato K, Ceresi E, Yamada K, Machover D, Breard J, Mathe G: Establishment and characterization of a new human eosinophilic leukemia cell line. Blood 1985, 66:1233-1240.

45. Tsujimura T, Furitsu T, Morimoto M, Isozaki K, Nomura S, Matsuzawa Y, Kitamura Y, Kanakura Y: Ligand-independent activation of c-kit receptor tyrosine kinase in a murine mastocytoma cell line P-815 generated by a point mutation. Blood 1994, 83:2619-2626.

46. Furitsu $T$, Tsujimura $T$, Tono $T$, Ikeda $H$, Kitayama $H$, Koshimizu U, Sugahara $\mathrm{H}$, Butterfield JH, Ashman LK, Kanayama Y, et al: Identification of mutations in the coding sequence of the proto-oncogene c-kit in a human mast cell leukemia cell line causing ligand-independent activation of c-kit product. J Clin Invest 1993, 92:1736-1744.

47. Heinrich MC, Griffith DJ, Druker BJ, Wait CL, Ott KA, Zigler AJ: Inhibition of c-kit receptor tyrosine kinase activity by STI 571, a selective tyrosine kinase inhibitor. Blood 2000, 96:925-932.

48. Bauer S, Yu LK, Demetri GD, Fletcher JA: Heat shock protein 90 inhibition in imatinib-resistant gastrointestinal stromal tumor. Cancer Res 2006, 66:9153-9161.

49. Tuveson DA, Willis NA, Jacks T, Griffin JD, Singer S, Fletcher CD, Fletcher JA Demetri GD: STI571 inactivation of the gastrointestinal stromal tumor cKIT oncoprotein: biological and clinical implications. Oncogene 2001, 20:5054-5058.

50. Yee KW, O'Farrell AM, Smolich BD, Cherrington JM, McMahon G, Wait CL, McGreevey LS, Griffith DJ, Heinrich MC: SU5416 and SU5614 inhibit kinase activity of wild-type and mutant FLT3 receptor tyrosine kinase. Blood 2002, 100:2941-2949.

51. Heinrich MC, Hoatlin ME, Zigler AJ, Silvey KV, Bakke AC, Keeble WW, Zhi Y, Reifsteck CA, Grompe M, Brown MG, et al: DNA cross-linker-induced G2/M arrest in group C Fanconi anemia lymphoblasts reflects normal checkpoint function. Blood 1998, 91:275-287.

52. Schittenhelm MM, Yee KW, Tyner JW, McGreevey L, Haley AD, Town A, Griffith DJ, Bainbridge T, Braziel RM, O'Farrell AM, et al: FLT3 K663Q is a novel AML-associated oncogenic kinase: Determination of biochemical properties and sensitivity to Sunitinib (SU11248). Leukemia 2006, 20:2008-2014.

doi:10.1186/1476-4598-12-19

Cite this article as: Kampa-Schittenhelm et al:: Quizartinib (AC220) is a potent second generation class III tyrosine kinase inhibitor that displays a distinct inhibition profile against mutant-FLT3, -PDGFRA and -KIT isoforms. Molecular Cancer 2013 12:19. 